\section{OPEN ACCESS}

Edited by:

Shamik Sen,

Indian Institute of Technology

Bombay, India

Reviewed by:

Takashi Miura,

Kyushu University, Japan

Srikala Raghavan,

Institute for Stem Cell Science and

Regenerative Medicine (InStem), India

*Correspondence:

Elie El Agha

elie.el-agha@

innere.med.uni-giessen.de

Specialty section:

This article was submitted to

Cell Adhesion and Migration,

a section of the journal

Frontiers in Cell and Developmental

Biology

Received: 30 October 2020

Accepted: 18 December 2020

Published: 14 January 2021

Citation:

Kina YP, Khadim A, Seeger W and EI Agha $E$ (2021) The Lung Vasculature:

A Driver or Passenger in Lung

Branching Morphogenesis?

Front. Cell Dev. Biol. 8:623868.

doi: $10.3389 /$ fcell.2020.623868

\title{
The Lung Vasculature: A Driver or Passenger in Lung Branching Morphogenesis?
}

\section{Yelda Pakize Kina, Ali Khadim, Werner Seeger and Elie El Agha*}

Department of Internal Medicine, Universities of Giessen and Marburg Lung Center (UGMLC), Institute for Lung Health (ILH), Cardio-Pulmonary Institute (CPI), Member of the German Center for Lung Research (DZL), Justus-Liebig University Giessen, Giessen, Germany

Multiple cellular, biochemical, and physical factors converge to coordinate organogenesis. During embryonic development, several organs such as the lung, salivary glands, mammary glands, and kidneys undergo rapid, but intricate, iterative branching. This biological process not only determines the overall architecture, size and shape of such organs but is also a pre-requisite for optimal organ function. The lung, in particular, relies on a vast surface area to carry out efficient gas exchange, and it is logical to suggest that airway branching during lung development represents a rate-limiting step in this context. Against this background, the vascular network develops in parallel to the airway tree and reciprocal interaction between these two compartments is critical for their patterning, branching, and co-alignment. In this mini review, we present an overview of the branching process in the developing mouse lung and discuss whether the vasculature plays a leading role in the process of airway epithelial branching.

Keywords: branching morphogenesis, endothelium, VEGF-vascular endothelial growth factor, VEGFR-vascular endothelial growth factor receptor, lung

\section{INTRODUCTION}

Cellular rearrangement and pattern formation are integral parts of organogenesis. Cell-cell communication, particularly between the endoderm-derived epithelium and the surrounding mesoderm-derived mesenchyme, sets the stage for lung formation. Such epithelial-mesenchymal interactions are highly defined along the proximal-distal axis in the developing lung. For example, it has been shown that grafting distal lung mesenchyme at the level of the tracheal epithelium leads to ectopic budding and subsequent branching at the grafting site (Alescio and Cassini, 1962). Such bronchial mesoderm, but not non-specific mesoderm, has been shown to be required for branching of the endoderm (Spooner and Wessells, 1970). Additionally, different thresholds of mesenchymal cell abundance have been shown to be required for either epithelial maintenance (in the case of minimal mesenchymal mass), or growth, morphogenesis and differentiation (in the case of larger mesenchymal mass) (Masters, 1976). Later, it was shown that the distal lung mesenchyme is characterized by the expression of fibroblast growth factor 10 ( Fgflo) (Bellusci et al., 1997) and that Fgf10-knockout embryos suffer from lung agenesis as well as other morphogenic and organogenic abnormalities (Min et al., 1998; Sekine et al., 1999; Suzuki et al., 2000; Sakaue et al., 2002; Jaskoll et al., 2005). In agreement with the study mentioned above regarding the role of the mesenchymal mass (Masters, 1976), it was shown that Fgf10 dosage is critical for 
the amplification of epithelial progenitors during embryonic lung development (Ramasamy et al., 2007).

Lung development starts with the evagination of the ventral foregut endoderm at embryonic day 9.5 (E9.5) to form the primitive lung buds, thus marking the embryonic stage of lung development that lasts until E12.5 (Figure 1). The lung domain in the foregut is marked by the expression of the transcription factor $N k x 2$.1. Surrounded by the splanchnic mesoderm, the two lung buds start undergoing iterative branching, the hallmark feature of the pseudoglandular stage of lung development (E12.5E16.5) (Figure 1). Other stages of lung development include the canalicular (E16.5-E17.5), saccular (E17.5 to post-natal day 5; P5), and alveolar stages (P5-P30) (Figure 1). The nomenclature of these stages is mainly based on the histological appearance of these lungs when analyzed by light microscopy (Warburton et al., 2010; El Agha and Bellusci, 2014; Volckaert and De Langhe, 2015).

The mesenchyme is a multifaceted compartment of the developing lung containing a heterogenous mixture of progenitor and differentiated cells, and it represents a reservoir of key growth factors, signaling networks, and even physical forces that direct lung development (Warburton et al., 2005, 2010; Morrisey and Hogan, 2010; El Agha and Bellusci, 2014; McCulley et al., 2015). Mesenchyme topology and dynamics in terms of cellular density and orientation around the growing epithelial buds likely guides the process of morphogenesis, particularly the branching process. Apart from FGF10+ mesenchymal cells that also act as progenitors for differentiated mesenchymal cells such as airway and vascular smooth muscle cells and lipofibroblasts (Mailleux et al., 2005; El Agha et al., 2014, 2017; Al Alam et al., 2015), airway smooth muscle cells (Kim et al., 2015) and nerve cells (Bower et al., 2014; Rhodes et al., 2015) have been proposed to be critical for the branching process, although the involvement of airway smooth muscle cell peristalsis in promoting epithelial branching in vivo has been recently questioned (Young et al., 2020) (Figure 1). Wingless-related integration site (WNT) signaling and extracellular matrix (ECM) deposition such as fibronectinThe latter is believed to act as "a rock in the stream" that forces the bifurcation of the growing epithelial tubes (Warburton et al., 2005) - have also been proposed as main drivers of epithelial branching (De Langhe et al., 2005; Kadzik et al., 2014) (Figure 1).

Physical forces have also emerged as an important regulator of epithelial branching (Figure 1). In fact, lung branching has been proposed to be a natural physical consequence of the interaction between cellular layers with surface tension between them (Lubkin and Murray, 1995). Several mathematical models for branching morphogenesis have been proposed such as diffusion-limited growth by FGF (Miura and Shiota, 2002), Turing instability-based model (Menshykau et al., 2012) and mixed-type models (Guo et al., 2014a,b). These models have been reviewed in Miura (2015). Interestingly, it has been shown that increased intraluminal pressure by tracheal occlusion, achieved via cauterization, boosts epithelial branching in air-liquid interface (ALI) cultures of E12.5 embryonic lungs (Unbekandt et al., 2008). Gene expression analysis showed that several key growth factors such as Fgf10, sonic hedgehog (Shh), and vascular endothelial growth factor a $(V e g f a)$ were upregulated whereas sprouty 2 (Spry2), a downstream target of FGF10 and an inhibitor of FGF signaling, was downregulated after $48 \mathrm{~h}$ of culture (Unbekandt et al., 2008).

During lung development, the vascular tree develops in parallel to its epithelial counterpart where proximal vessels, formed through the process of angiogenesis, and distal vessels, formed through the process of vasculogenesis, fuse to form a continuous vascular lumen around E13/E14 (deMello et al., 1997). The lung vasculature is involved in the regulation of many processes during lung development and its involvement in epithelial branching has been a topic of extensive research. Here, we discuss the various modes of lung branching and whether the lung vasculature guides branching morphogenesis of the lung epithelium during early lung development.

\section{THE BRANCHING PROGRAM OF THE DEVELOPING MOUSE LUNG: HARD-WIRED AND STOCHASTIC ASPECTS}

Congenital diaphragmatic hernia $(\mathrm{CDH})$ is a defect resulting from impaired development, and therefore incomplete closure, of the diaphragm, leading to the invasion of abdominal organs into the thoracic cavity. $\mathrm{CDH}$ newborns suffer from lung hypoplasia and the experimental model of nitrofen-induced $\mathrm{CDH}$ in rodents has shown that epithelial branching is indeed impaired in these embryos (Guilbert et al., 2000). This defect therefore favors a model where available space dictates the extent of lung branching. However, the assumption that lung branching morphogenesis is an iterative process that proceeds in a random fashion until the intrathoracic space is filled has already been challenged. Members of various developmental signaling pathways such as FGF, SHH, bone morphogenetic protein (BMP), and WNT signaling have been implicated in this process (Cardoso and $\mathrm{Lu}$, 2006; Warburton et al., 2010) (Figure 1). In a pioneering study, the branch lineage of the bronchial tree and the accompanying branching sequences were analyzed between E11 and E15 using fixed specimens (Metzger et al., 2008). Thorough analysis of such samples showed that the developing lung undergoes three modes of branching defined as domain branching, planar bifurcation, and orthogonal bifurcation. Domain branching sets up the overall shape of individual lobes and is controlled by two patterning systems: A proximal-distal system controlling periodicity/sequence of branching and a circumferential system specifying the position of domains and the order in which they are implemented. Planar bifurcation forms the edges of the lobes and therefore occurs at their tips while orthogonal bifurcation generates the surfaces of the lobes with a $90^{\circ}$ rotation in the bifurcation plane between each round of branching. These findings indicate that the branching process is predominantly stereotyped by genetic regulators although there are instances where anomalous branching events, defined as "branching errors" also take place. The branching pattern was therefore proposed to be controlled by a global master routine that controls further subroutines, setting the coupling scheme for each lineage 
Murine lung development

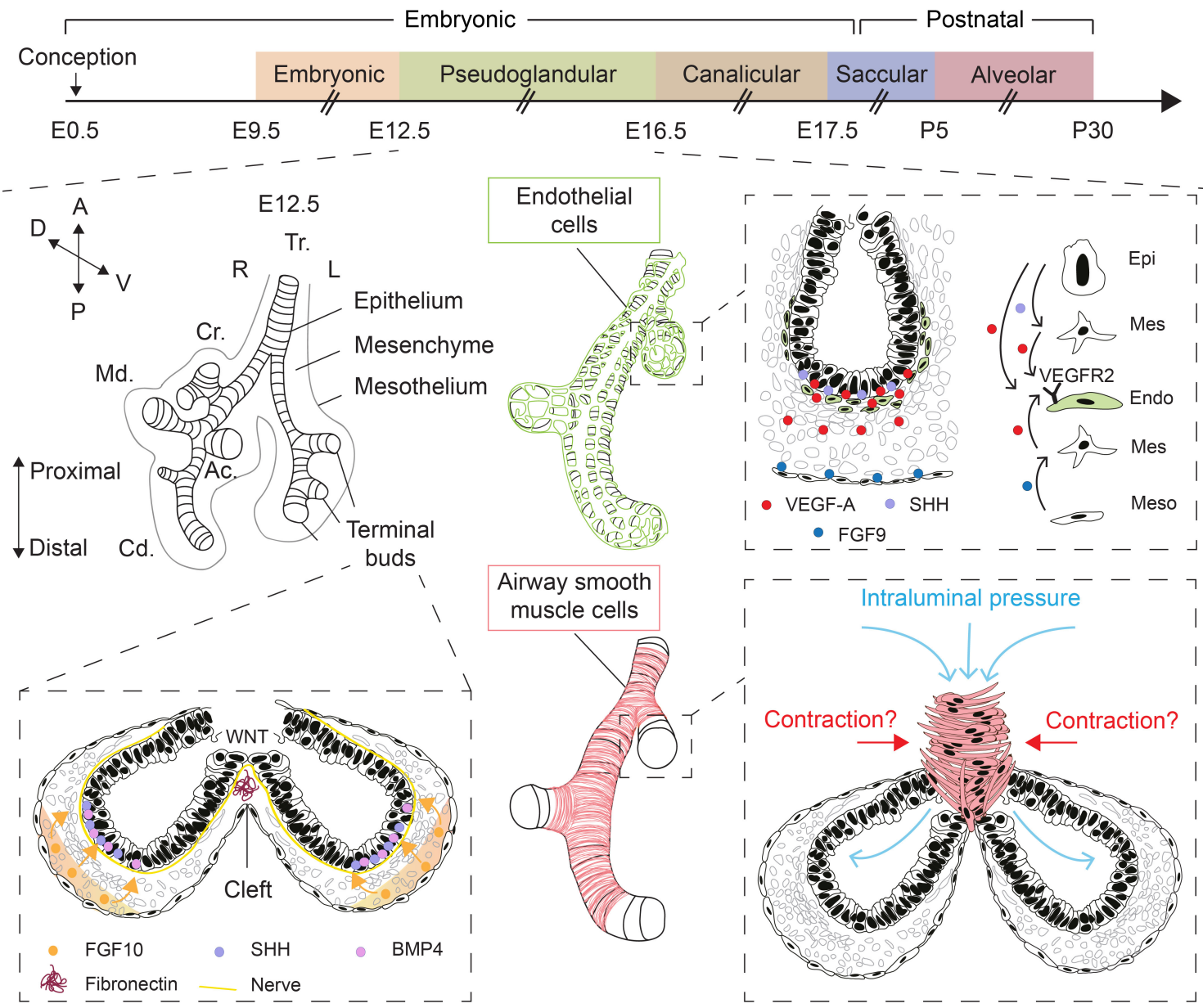

FIGURE 1 | Overview of lung development and various factors controlling epithelial branching morphogenesis. During the pseudoglandular stage of lung development, the lung undergoes successive rounds of branching. FGF10, secreted by submesothelial mesenchymal cells, induces budding and branching of distal terminal epithelial buds. WNT signaling in the distal epithelium induces the deposition of fibronectin at the cleft region, which helps guide bifurcation. Epithelial, mesenchymal, and mesothelial cells cooperate to promote the development of the endothelial network through VEGF-AVEGFR2 signaling. Intraluminal pressure, innervation, and airway smooth muscle cell differentiation are among many factors that promote branching morphogenesis. Orientation vector: A, Anterior; P, Posterior; D, Dorsal; V, Ventral. Embryonic lung anatomy: Tr, Trachea; R, Right; L, Left; Cr, Cranial lobe; Md, Medial lobe; Cd, Caudal lobe; Ac, Accessory lobe. Cell-type code: Endo, Endothelium; Epi, Epithelium; Mes, Mesenchyme; Meso, Mesothelium. The timeline representation of murine lung development does not correspond to a fixed scale.

early, and then relaxing and allowing these lineages to continue independently (Metzger et al., 2008).

The paradigm that lung branching morphogenesis is stereotyped and hard-wired was challenged by Blanc et al. (2012) who carried out three-dimensional (3D) reconstruction of whole right cranial lobes between E11.25 and E13.5. The authors concluded that beyond early branching generations, the branching stereotypy loosens up, and epithelial buds simply try to homogenously fill the surrounding mesenchymal space. The dynamic changes of mesodermal shape seemed to influence both the branching pattern and branching rate, indicating that branching of the endoderm is basically connected to local changes of mesoderm growth (Blanc et al., 2012). Those findings indicated that the branching mode of the epithelial tree is unlikely to be specified and predefined by a rigid, global genetic program.

\section{VEGF-A/VEGFR2 SIGNALING POSITIVELY REGULATES EPITHELIAL BRANCHING MORPHOGENESIS}

VEGFs are critical ligands for the development of blood vessels from hemangioblasts, a population of hematopoietic, and endothelial progenitor cells. These ligands are produced by both epithelial and mesenchymal cells at E12.5. Using 
organotypic cultures of E11.5 lung explants grown in an ALI for up to 4 days, it was shown that VEGF-A is deposited in the subepithelial mesenchyme adjacent to the branching distal epithelial buds where it upregulates endothelial cell markers and promotes vasculogenesis (Healy et al., 2000) (Figure 1). Such expression pattern hinted at a possible role for VEGF-A in coordinating airway branching and blood vessel formation during early lung development. Later work demonstrated that epithelium-derived $\mathrm{SHH}$ and mesotheliumderived FGF9 cooperate to induce Vegfa expression in both the subepithelial and submesothelial mesenchyme, that in turn acts on vascular endothelial growth factor receptor 2-positive (VEGFR2+; aka fetal liver kinase 1-positive or FLK1+) endothelial progenitors to induce vasculogenesis, thus contributing to the formation of the capillary plexus (White et al., 2007) (Figure 1). Interestingly, overexpression of Vegfa in distal epithelial cells, but not in proximal airways, disrupts the peripheral vascular network, and impairs epithelial branching (Akeson et al., 2003). The latter findings highlight the importance of the crosstalk between the epithelium and endothelium in the distal lung and that disturbance of the spatial and temporal control of VEGF-A signaling has implications for branching morphogenesis.

Since lung development proceeds in a hypoxic environment inside the uterus, it was hypothesized that low oxygen levels represent an important factor for lung branching morphogenesis as well as vascular development. Indeed, E11.5 embryonic lung explants grown in ALI at 3\% oxygen exhibited enhanced numbers of terminal epithelial branches compared with controls cultured at $20 \%$ oxygen (van Tuyl et al., 2005). Interestingly, the spatial expression patterns of mesenchymal Fgf10 and epithelial Bmp4 did not seem to be affected by hypoxia. Moreover, vascular development was boosted by hypoxia as evident by the extensive endothelial network that extended from the trachea to distal epithelial tips. Knock down of Hifla and Vegfa led to dramatic impairment of vascularization in these lung explants while exogenous VEGF treatment rescued the phenotype in Vegfa-, but not Hif1a-, knockdown explants. Importantly, arrested vascular development coincided with significant simplification of the epithelial tree indicating that the disturbance of vascular network assembly disrupts epithelial branching (van Tuyl et al., 2005).

The positive effect of VEGF signaling on epithelial branching has been shown to be mediated by endothelial-epithelial crosstalk (Gebb and Shannon, 2000; Del Moral et al., 2006). Using organotypic cultures of E11.5 lung explants, it was shown that treatment with VEGF enhanced the number of terminal epithelial branches in parallel to increased epithelial and mesenchymal proliferation (Del Moral et al., 2006). VEGF did not have a direct effect on isolated endoderm. The impact on lung branching coincided with the upregulation of Flk1, Sftpc, and Bmp4 and the downregulation of Spry2/4, which are known inhibitors of FGF signaling. Conversely, knock down of Flk1 led to reduced terminal branching with downregulation of Flk1, $S f t p c$, and Bmp4 in parallel to upregulation of Spry2/4. Loss of Flk1 expression also led to significant reduction of proliferation in both the epithelium and mesenchyme (Del Moral et al., 2006). Those findings suggested that the vasculature is an important factor for promoting epithelial branching during early lung development.

In another study, the effect of in vivo vascular depletion on the branching stereotypy and the coordination between the branching of epithelial and vascular tubes leading to their coalignment was studied (Lazarus et al., 2011). A loss-of-function approach involving the overexpression of a dominant-negative, decoy vascular endothelial growth factor receptor 1 (VEGFR1) to inhibit the effect of VEGF signaling between E6.5 and E12.5 was employed. E12.5 lungs showed that vascular ablation caused fewer, more dilated airway branches in addition to ectopic branching events compared with control lungs (Lazarus et al., 2011). This was accompanied by upregulation of Spry2 and downregulation of Shh, the gene encoding its receptor (patched 1 or Ptc1) and downstream target glioma-associated oncogene 1 (Gli1). Importantly, domain branches, as described by Metzger et al. (2008), requiring a change in the branching plane were preferentially affected by vascular depletion. These branching abnormalities coincided with impaired expression of the branching mediator FgflO and branching regulators Shh and Spry2 (Lazarus et al., 2011). Additionally, treatment of E11.5 ALI cultures with a VEGFR2 intracellular inhibitor led to vascular depletion coupled to a $30 \%$ reduction in planar bifurcations and a $70 \%$ reduction in orthogonal bifurcations (Lazarus et al., 2011). Remarkably, the in vivo experiments showed that airway branching and the $3 \mathrm{D}$ architecture of the lung seemed to be fully rescued as a consequence of revascularization due to termination of VEGF blockade between E12.5 and postnatal day 14 (P14), indicating that the branching arrest was reversible and that vascular regain was at least permissive for kick-starting epithelial branching morphogenesis (Lazarus et al., 2011).

Using organoid cultures, it was shown that human umbilical vein endothelial cells (HUVECs) facilitated the generation of branched bronchioalveolar organoids from human bronchial epithelial cells (Franzdóttir et al., 2010). Further analysis confirmed that airway epithelial cells were polarized, and that inhibition of FGF signaling via pharmacological intervention inhibited the branching process (Franzdóttir et al., 2010).

Another culture system involves the engraftment of E12.5 lungs under the kidney capsule of adult host mice. Using this model, it was shown that inhibiting VEGF-A activity by injecting host animals with a dominantnegative decoy mFLT(1-3)Ig led to the inhibition of both vascular development and epithelial branching (Zhao et al., 2005).

Collectively, there is strong evidence suggesting that the vascular network is critical for proper epithelial morphogenesis, at least in part by affecting the spatial expression pattern of key genes involved in the branching process. In the next section, evidence suggesting that the lung epithelium is capable of undergoing branching morphogenesis in the absence of the vascular system will be presented. 


\section{THE LUNG EPITHELIUM IS CAPABLE OF BRANCHING IN THE ABSENCE OF ENDOTHELIAL CELLS}

Despite the general notion that the lung vasculature has a strong influence on epithelial branching in the developing lung, several lines of evidence suggest that the vasculature is dispensable for epithelial branching. For example, it has been reported that epithelial branching proceeds even in the absence of endothelial cells in vitro (Havrilak and Shannon, 2015). The authors used ALI cultures of E12.5 lung explants to show that treatment with various VEGFR inhibitors did not impair the branching process after $48 \mathrm{~h}$ of ex vivo culture. Loss of endothelial cells as a result of such treatment did not seem to impair epithelial, smooth muscle or pericyte differentiation. Additionally, the authors elegantly showed that distal mesenchymal cell suspensions that had been depleted of endothelial cells were capable of promoting the formation of branched structures when combined with distal epithelial cell suspensions and allowed to grow on a membrane for 5-7 days in the presence of SHH stimulation (Havrilak and Shannon, 2015). Moreover, they also showed that primary lung endothelial cells isolated from E12.5 lungs or human lung microvascular endothelial cells (HMVEC-L) were not capable of promoting branching of epithelial rudiments when cultured in basement membrane extract as opposed to the positive control cultured in the appropriate medium (Havrilak and Shannon, 2015). Those findings argue that the endothelial component is dispensable for epithelial morphogenesis and branching. Followup work showed that pharmacological inhibition of VEGFR at E8.5 did not affect early lung specification and bud formation (Havrilak et al., 2017). On the other hand, analysis of Flk1knockout embryos that do not develop endothelial cells revealed impaired pulmonary specification in the mutant endoderm likely due to delayed development as such mutants were able to catch up with the controls in terms of respiratory specification and bud formation when cultured ex vivo (Havrilak et al., 2017).

Another line of evidence for the dispensability of endothelial cells for lung branching comes from the organoid field. Human pluripotent stem cells (hPSCs) can be used to generate endoderm spheroids, and further conditioned to give rise to branched lung organoids in the absence of endothelial cells (Miller et al., 2019). Even earlier studies using isolated endoderm have shown that recombinant growth factors such as FGF1 or FGF10 are sufficient to induce epithelial branching (Nogawa and Ito, 1995; Bellusci et al., 1997). Co-culturing bronchioalveolar stem cells (BASCs) characterized by dual expression of airway and alveolar markers (Scgbla1 and Sftpc, respectively) with appropriate resident mesenchymal cells (CD45- CD31- EpCAM- SCA-1+) leads to the formation of highly branched bronchioalveolar organoids that are reminiscent of the branching lung (VazquezArmendariz et al., 2020). Last but not least, several theoretical works have shown that FGFs per se drive lung branching (Miura and Shiota, 2002; Clément et al., 2012). These data, as well as others, strongly suggest that the epithelium is highly responsive to mesenchymal signals that instruct branching morphogenesis and that the vasculature does not seem to play a leading role nor is absolutely required for epithelial branching.

\section{DISCUSSION}

Tissue interactions represent a hallmark feature of lung organogenesis, and various cellular, molecular, biochemical, and physical mechanisms have been implicated in this developmental process. In this context, the interplay between epithelial, mesenchymal, mesothelial, and endothelial cells is absolutely critical for coordinating the development of the various constituent tissues within the lung. As highlighted above, vascular ablation in vivo mainly disrupts vascular development but also perturbs epithelial branching patterns. The observation that endothelial cells appear to be dispensable for epithelial rearrangement and branching suggests that the vascular system seems to play a modulatory role and is mainly involved in downstream patterning of airway branching during the course of lung development. The influence of the lung endothelium might be mediated by endothelium-derived factors that modulate ECM properties such as stiffness or heparan sulfate proteoglycan composition. Heparan sulfates are known to interact with diffusible proteins such as FGF10 and create growth factor gradients that instruct epithelial patterning (Izvolsky et al., 2003; Warburton et al., 2010). Therefore, it is plausible that the endothelium creates a permissive or modulatory environment that impacts branching patterns along the growing epithelial tubes. Given the close association between the distal epithelium and the endothelial layer, it is possible that interaction between the two cellular domains across the basement membrane is mediated by paracrine-acting growth factors (other than VEGF-A/VEGFR2 signaling) and downstream (positive and negative) feedback-loop mechanisms. Epithelial-endothelial interaction might also be mediated by ECM-integrin signaling or even physical forces arising from sprouting of endothelial cells leading to the engulfment of terminal buds by the capillary plexus.

Despite the widespread use of the elegant ALI organotypic culture system, one of its limitations is that it heavily distorts the $3 \mathrm{D}$ structure of embryonic lungs and leads to severe flattening on polycarbonate membranes. This issue might mask potential deleterious effects for vascular ablation on the 3D patterning of epithelial branches. Moreover, oxygen concentration, the absence of the systemic blood circulation and extrapulmonary cells such as bone marrow-derived cells and loss of nerve connections collectively alter the physiological, biochemical and biophysical context, and certainly do not mimic the in-utero environment. On the other hand, the majority of the in vivo genetic approaches that have so far been employed do not perturb the endothelium in a cellautonomous fashion, which opens the door for potential nonspecific effects on non-endothelial cells. Future studies designed to selectively manipulate/ablate endothelial cells in the lung during defined intervals with minimal off-target effects in vivo might unveil novel mechanisms related to epithelial-endothelial 
crosstalk along the proximal-distal axis and their impact on lung branching.

\section{AUTHOR CONTRIBUTIONS}

YK, AK, WS, and EEA drafted and edited the manuscript. All authors contributed to the article and approved the submitted version.

\section{REFERENCES}

Akeson, A. L., Greenberg, J. M., Cameron, J. E., Thompson, F. Y., Brooks, S. K., Wiginton, D., et al. (2003). Temporal and spatial regulation of VEGF-A controls vascular patterning in the embryonic lung. Dev. Biol. 264, 443-455. doi: 10.1016/j.ydbio.2003.09.004

Al Alam, D., El Agha, E., Sakurai, R., Kheirollahi, V., Moiseenko, A., Danopoulos, S., et al. (2015). Evidence for the involvement of fibroblast growth factor 10 in lipofibroblast formation during embryonic lung development. Development 142, 4139-4150. doi: 10.1242/dev.109173

Alescio, T., and Cassini, A. (1962). Induction in vitro of tracheal buds by pulmonary mesenchyme grafted on tracheal epithelium. J. Exp. Zool. 150, 83-94. doi: 10.1002/jez.1401500202

Bellusci, S., Grindley, J., Emoto, H., Itoh, N., and Hogan, B. L. (1997). Fibroblast growth factor 10 (FGF10) and branching morphogenesis in the embryonic mouse lung. Development 124, 4867-4878.

Blanc, P., Coste, K., Pouchin, P., Azaïs, J.-M., Blanchon, L., Gallot, D., et al. (2012). A role for mesenchyme dynamics in mouse lung branching morphogenesis. PLoS ONE 7:e41643. doi: 10.1371/journal.pone.0041643

Bower, D. V., Lee, H.-K., Lansford, R., Zinn, K., Warburton, D., Fraser, S. E., et al. (2014). Airway branching has conserved needs for local parasympathetic innervation but not neurotransmission. BMC Biol. 12:92. doi: 10.1186/s12915-014-0092-2

Cardoso, W. V., and Lu, J. (2006). Regulation of early lung morphogenesis: questions, facts and controversies. Development 133, 1611-1624. doi: $10.1242 /$ dev.02310

Clément, R., Douady, S., and Mauroy, B. (2012). Branching geometry induced by lung self-regulated growth. Phys. Biol. 9:066006. doi: 10.1088/1478-3975/9/6/066006

De Langhe, S. P., Sala, F. G., Del Moral, P. M., Fairbanks, T. J., Yamada, K. M., Warburton, D., et al. (2005). Dickkopf-1 (DKK1) reveals that fibronectin is a major target of Wnt signaling in branching morphogenesis of the mouse embryonic lung. Dev. Biol. 277, 316-331. doi: 10.1016/j.ydbio.2004.09.023

Del Moral, P. M., Sala, F. G., Tefft, D., Shi, W., Keshet, E., Bellusci, S., et al. (2006). VEGF-A signaling through Flk-1 is a critical facilitator of early embryonic lung epithelial to endothelial crosstalk and branching morphogenesis. Dev. Biol. 290, 177-188. doi: 10.1016/j.ydbio.2005.11.022

deMello, D. E., Sawyer, D., Galvin, N., and Reid, L. M. (1997). Early fetal development of lung vasculature. Am. J. Respir. Cell Mol. Biol. 16, 568-581. doi: 10.1165/ajrcmb.16.5.9160839

El Agha, E., and Bellusci, S. (2014). Walking along the fibroblast growth factor 10 route: a key pathway to understand the control and regulation of epithelial and mesenchymal cell-lineage formation during lung development and repair after injury. Scientifica 2014:538379. doi: 10.1155/2014/53 8379

El Agha, E., Herold, S., Al Alam, D., Quantius, J., MacKenzie, B., Carraro, G., et al. (2014). Fgf10-positive cells represent a progenitor cell population during lung development and postnatally. Development 141, 296-306. doi: 10.1242/dev.09 9747

El Agha, E., Kheirollahi, V., Moiseenko, A., Seeger, W., and Bellusci, S. (2017). Ex vivo analysis of the contribution of FGF10+ cells to airway smooth muscle cell formation during early lung development. Dev. Dyn. 246, 531-538. doi: 10.1002/dvdy.2 4504

\section{FUNDING}

EEA was funded by the Institute for Lung Health (ILH) and grants from the German Research Foundation (DFG, KFO309 P7, and SFB CRC1213-project A04), Excellence Cluster CardioPulmonary System (ECCPS), and University Hospital Giessen and Marburg (UKGM). EEA also acknowledges the support of the German Center for Lung Research (DZL) and the CardioPulmonary Institute (CPI, EXC 2026, Project ID: 390649896).

Franzdóttir, S. R., Axelsson, I. T., Arason, A. J., Baldursson, Ó., Gudjonsson, T., and Magnusson, M. K. (2010). Airway branching morphogenesis in three dimensional culture. Respir. Res. 11:162. doi: 10.1186/1465-9921-11-162

Gebb, S. A., and Shannon, J. M. (2000). Tissue interactions mediate early events in pulmonary vasculogenesis. Dev. Dyn. 217, 159-169. doi: 10.1002/(SICI)10970177(200002)217:2<159::AID-DVDY3>3.0.CO;2-9

Guilbert, T. W., Gebb, S. A., and Shannon, J. M. (2000). Lung hypoplasia in the nitrofen model of congenital diaphragmatic hernia occurs early in development. Am. J. Physiol. Lung Cell. Mol. Physiol. 279, L1159-L1171. doi: 10.1152/ajplung.2000.279.6.L1159

Guo, Y., Chen, T.-H., Zeng, X., Warburton, D., Boström, K. I., Ho, C.-M., et al. (2014a). Branching patterns emerge in a mathematical model of the dynamics of lung development. J. Physiol. 592, 313-324. doi: 10.1113/jphysiol.2013.261099

Guo, Y., Sun, M., Garfinkel, A., and Zhao, X. (2014b). Mechanisms of side branching and tip splitting in a model of branching morphogenesis. PLoS ONE 9:e102718. doi: 10.1371/journal.pone.0102718

Havrilak, J. A., Melton, K. R., and Shannon, J. M. (2017). Endothelial cells are not required for specification of respiratory progenitors. Dev. Biol. 427, 93-105. doi: 10.1016/j.ydbio.2017.05.003

Havrilak, J. A., and Shannon, J. M. (2015). Branching of lung epithelium in vitro occurs in the absence of endothelial cells. Dev. Dyn. 244, 553-563. doi: $10.1002 /$ dvdy.24251

Healy, A. M., Morgenthau, L., Zhu, X., Farber, H. W., and Cardoso, W. V. (2000). VEGF is deposited in the subepithelial matrix at the leading edge of branching airways and stimulates neovascularization in the murine embryonic lung. Dev. Dyn. 219, 341-352. doi: 10.1002/1097-0177(2000)9999:9999<::AIDDVDY1061>3.0.CO;2-M

Izvolsky, K. I., Zhong, L., Wei, L., Yu, Q., Nugent, M. A., and Cardoso, W. V. (2003). Heparan sulfates expressed in the distal lung are required for Fgf10 binding to the epithelium and for airway branching. Am. J. Physiol. Lung Cell. Mol. Physiol. 285, L838-L846. doi: 10.1152/ajplung.00081.2003

Jaskoll, T., Abichaker, G., Witcher, D., Sala, F. G., Bellusci, S., Hajihosseini, M. K., et al. (2005). FGF10/FGFR2b signaling plays essential roles during in vivo embryonic submandibular salivary gland morphogenesis. BMC Dev. Biol. 5:11. doi: 10.1186/1471-213X-5-11

Kadzik, R. S., Cohen, E. D., Morley, M. P., Stewart, K. M., Lu, M. M., and Morrisey, E. E. (2014). Wnt ligand/frizzled 2 receptor signaling regulates tube shape and branch-point formation in the lung through control of epithelial cell shape. Proc. Nat. Acad. Sci. U.S.A. 111, 12444-12449. doi: 10.1073/pnas.1406639111

Kim, H. Y., Pang, M.-F., Varner, V. D., Kojima, L., Miller, E., Radisky, D. C., et al. (2015). Localized smooth muscle differentiation is essential for epithelial bifurcation during branching morphogenesis of the mammalian lung. Dev. Cell 34, 719-726. doi: 10.1016/j.devcel.2015.08.012

Lazarus, A., Del-Moral, P. M., Ilovich, O., Mishani, E., Warburton, D., and Keshet, E. (2011). A perfusion-independent role of blood vessels in determining branching stereotypy of lung airways. Development 138, 2359-2368. doi: 10.1242/dev.060723

Lubkin, S. R., and Murray, J. D. (1995). A mechanism for early branching in lung morphogenesis. J. Math. Biol. 34, 77-94. doi: 10.1007/BF00180137

Mailleux, A. A., Kelly, R., Veltmaat, J. M., De Langhe, S. P., Zaffran, S., Thiery, J. P., et al. (2005). Fgf10 expression identifies parabronchial smooth muscle cell progenitors and is required for their entry into the smooth muscle cell lineage. Development 132, 2157-2166. doi: 10.1242/dev.01795 
Masters, J. R. W. (1976). Epithelial-mesenchymal interaction during lung development: the effect of mesenchymal mass. Dev. Biol. 51, 98-108. doi: 10.1016/0012-1606(76)90125-1

McCulley, D., Wienhold, M., and Sun, X. (2015). The pulmonary mesenchyme directs lung development. Curr. Opin. Genet. Dev. 32, 98-105. doi: 10.1016/j.gde.2015.01.011

Menshykau, D., Kraemer, C., and Iber, D. (2012). Branch mode selection during early lung development. PLoS Comput. Biol. 8:e1002377. doi: 10.1371/journal.pcbi.1002377

Metzger, R. J., Klein, O. D., Martin, G. R., and Krasnow, M. A. (2008). The branching programme of mouse lung development. Nature 453, 745-750. doi: $10.1038 /$ nature 07005

Miller, A. J., Dye, B. R., Ferrer-Torres, D., Hill, D. R., Overeem, A. W., Shea, L. D., et al. (2019). Generation of lung organoids from human pluripotent stem cells in vitro. Nat. Protoc. 14, 518-540. doi: 10.1038/s41596-018-0104-8

Min, H., Danilenko, D. M., Scully, S. A., Bolon, B., Ring, B. D., Tarpley, J. E., et al. (1998). Fgf-10 is required for both limb and lung development and exhibits striking functional similarity to drosophila branchless. Genes Dev. 12, 3156-3161. doi: 10.1101/gad.12.20.3156

Miura, T. (2015). Models of lung branching morphogenesis. J. Biochem. 157, 121-127. doi: 10.1093/jb/mvu087

Miura, T., and Shiota, K. (2002). Depletion of FGF acts as a lateral inhibitory factor in lung branching morphogenesis in vitro. Mech. Dev. 116, 29-38. doi: 10.1016/S0925-4773(02)00132-6

Morrisey, E. E., and Hogan, B. L. (2010). Preparing for the first breath: genetic and cellular mechanisms in lung development. Dev. Cell 18, 8-23. doi: 10.1016/j.devcel.2009.12.010

Nogawa, H., and Ito, T. (1995). Branching morphogenesis of embryonic mouse lung epithelium in mesenchyme-free culture. Development 121, 1015-1022.

Ramasamy, S. K., Mailleux, A. A., Gupte, V. V., Mata, F., Sala, F. G., Veltmaat, J. M., et al. (2007). Fgf10 dosage is critical for the amplification of epithelial cell progenitors and for the formation of multiple mesenchymal lineages during lung development. Dev. Biol. 307, 237-247. doi: 10.1016/j.ydbio.2007.0 4.033

Rhodes, J., Saxena, D., Zhang, G. F., Gittes, G. K., and Potoka, D. A. (2015). Defective parasympathetic innervation is associated with airway branching abnormalities in experimental CDH. Am. J. Physiol. Lung Cell. Mol. Physiol. 309, L168-L174. doi: 10.1152/ajplung.00299. 2014

Sakaue, H., Konishi, M., Ogawa, W., Asaki, T., Mori, T., Yamasaki, M., et al. (2002). Requirement of fibroblast growth factor 10 in development of white adipose tissue. Genes Dev. 16, 908-912. doi: 10.1101/gad.983202

Sekine, K., Ohuchi, H., Fujiwara, M., Yamasaki, M., Yoshizawa, T., Sato, T., et al. (1999). Fgf10 is essential for limb and lung formation. Nat. Genet. 21, 138-141. doi: $10.1038 / 5096$

Spooner, B. S., and Wessells, N. K. (1970). Mammalian lung development: interactions in primordium formation and bronchial morphogenesis. J. Exp. Zool. 175, 445-454. doi: 10.1002/jez.1401750404
Suzuki, K., Yamanishi, K., Mori, O., Kamikawa, M., Andersen, B., Kato, S., et al. (2000). Defective terminal differentiation and hypoplasia of the epidermis in mice lacking the Fgf10 gene. FEBS Lett. 481, 53-56. doi: 10.1016/S0014-5793(00)01968-2

Unbekandt, M., Del Moral, P. M., Sala, F. G., Bellusci, S., Warburton, D., and Fleury, V. (2008). Tracheal occlusion increases the rate of epithelial branching of embryonic mouse lung via the FGF10-FGFR2b-sprouty2 pathway. Mech. Dev. 125, 314-324. doi: 10.1016/j.mod.2007.10.013

van Tuyl, M., Liu, J., Wang, J., Kuliszewski, M., Tibboel, D., and Post, M. (2005). Role of oxygen and vascular development in epithelial branching morphogenesis of the developing mouse lung. Am. J. Physiol. Lung Cell. Mol. Physiol. 288, L167-178. doi: 10.1152/ajplung.00185.2004

Vazquez-Armendariz, A. I., Heiner, M., El Agha, E., Salwig, I., Hoek, A., Hessler, M. C., et al. (2020). Multilineage murine stem cells generate complex organoids to model distal lung development and disease. EMBO J. 39:e103476. doi: $10.15252 / \mathrm{embj} .2019103476$

Volckaert, T., and De Langhe, S. P. (2015). Wnt and FGF mediated epithelialmesenchymal crosstalk during lung development. Dev. Dyn. 244, 342-366. doi: $10.1002 /$ dvdy.24234

Warburton, D., Bellusci, S., De Langhe, S., Del Moral, P. M., Fleury, V., Mailleux, A., et al. (2005). Molecular mechanisms of early lung specification and branching morphogenesis. Pediatr. Res. 57, 26R-37R. doi: 10.1203/01.PDR.0000159570.01327.ED

Warburton, D., El-Hashash, A., Carraro, G., Tiozzo, C., Sala, F., Rogers, O., et al. (2010). Lung organogenesis. Curr. Top. Dev. Biol. 90, 73-158. doi: 10.1016/S0070-2153(10)90003-3

White, A. C., Lavine, K. J., and Ornitz, D. M. (2007). FGF9 and SHH regulate mesenchymal vegfa expression and development of the pulmonary capillary network. Development 134, 3743-3752. doi: 10.1242/dev.004879

Young, R. E., Jones, M. K., Hines, E. A., Li, R., Luo, Y., Shi, W., et al. (2020). Smooth muscle differentiation is essential for airway size, tracheal cartilage segmentation, but dispensable for epithelial branching. Dev. Cell 53, 73-85.e5. doi: 10.1016/j.devcel.2020.02.001

Zhao, L., Wang, K., Ferrara, N., and Vu, T. H. (2005). Vascular endothelial growth factor co-ordinates proper development of lung epithelium and vasculature. Mech. Dev. 122, 877-886. doi: 10.1016/j.mod.2005.04.001

Conflict of Interest: The authors declare that the research was conducted in the absence of any commercial or financial relationships that could be construed as a potential conflict of interest.

Copyright $\odot 2021$ Kina, Khadim, Seeger and El Agha. This is an open-access article distributed under the terms of the Creative Commons Attribution License (CC BY). The use, distribution or reproduction in other forums is permitted, provided the original author(s) and the copyright owner(s) are credited and that the original publication in this journal is cited, in accordance with accepted academic practice. No use, distribution or reproduction is permitted which does not comply with these terms. 